7 (2016)

DOI: $10.18276 /$ rk.2016.7-13

Łukasz Kołoczek

Uniwersytet Szczeciński

\title{
Die Transposition als eine Art Lektüre von Beiträge zur Philosophie
}

In diesem Artikel möchte ich die Frage stellen, wie die Lektüre der späten Heideggerschen Philosophie in anderer Sprache als Deutsch möglich ist. Weiter erkläre ich, warum diese Frage keine Randfrage ist, sondern warum und wie sie das Wesen der Philosophie von Heidegger betrifft. Meine Muttersprache ist Polnisch, deswegen gelten meine Erwägungen insbesondere der deutsch-polnischen Übersetzung, aber die Struktur meines Problems scheint universal zu sein und sie könnte wahrscheinlich bei jeder Sprache angewendet werden, in der man versuchen würde, die Werke von Heidegger zu lesen.

Heidegger selbst philosophiert, indem er eine Übersetzung macht. Er nennt zwar seine Arbeiten mal Kommentar, mal Erörterung, aber in jedem Fall, in dem der Ausgangspunkt seiner Erwägungen ein anderer Text ist, nehmen sie die Form einer Übersetzung an. Mir scheint, dass der Umstand - dessen Übersehen keine Schäden für das Verstehen der vielen wichtigen Werke von Heidegger verursacht - wesentlich ist für das Erfassen dessen, was der auf den Seiten von Beiträge zur Philosophie artikulierte und für spätere Werke dieses Philosophen grundlegende Entwurf des Seyns ist. Ohne Ansprüche auf Erklärung der ganzen Komplexität dieses Entwurfs, möchte ich die Behauptung wagen, dass dieser Entwurf eine Übersetzung ist. Eine merkwürdige Übersetzung, eine Übersetzung von etwas Besonderen.

Heidegger lässt im Prinzip nicht erkennen, wie er übersetzt. Es kann freilich an vielen Stellen beobachtet werden, wie die Übersetzung selbst „wirkt“, z.B. im Essay „Vom Wesen und Begriff der fisis“. Der Text ist eine Übersetzung des Fragments von Aristoteles Physik (des ersten Kapitels des zweiten Buches). Die Übersetzung setzt sich mit dem griechischen Text auseinander und gibt ihn auf Deutsch wider. Der übersetzte Text liegt aber fern von den verständlichen „philologischen“ Übersetzungen, und das Deutsch dieser Übersetzungen ist so unverständlich, dass sie eines 
begründenden und erklärenden Kommentars benötigen. Das Ziel dieses Vorgehens ist in diesem konkreten Fall offensichtlich, aber es verdeckt die Weise, auf die es erreicht wird. Heidegger erkennt, dass unter den Begriffen natura und fisis jeweils „eine Auslegung des Seienden im Ganzen“" (Heidegger, 1976: 240) versteckt ist, und die Physik von Aristoteles, „das verborgene und deshalb nie zureichend durchdachte Grundbuch der abendländischen Philosophie“, versteht er als „erstes denkerisch geschlossenes Begreifen der fisis“ (Heidegger, 1976: 242). Man kann also diesen Essay lesen, indem man versucht, den Grund der Metaphysik zu erreichen, ohne dabei zu vergessen, dass der Versuch den Charakter einer Übersetzung hat.

Es scheint, dass es im Heideggerschen Nachlass nicht viele Stellen gibt, an denen er berichten würde, wie er seine Vorgehensweise bei der Übersetzung versteht. Friedrich-Wilhelm von Herrmann zählt sie auf: 1. in der Abhandlung Der Spruch des Anaximander (1946) aus den Holzwegen; 2. in der zweiten Vorlesung Was heißt denken? aus dem Sommersemester 1953; 3. in der Vorlesung Der Satz vom Grund aus dem Wintersemester 1955/56 (Herrmann, 1994: 307). Aufgrund dieser Fragmente, und auch angeknüpft an andere Textstellen, zum Beispiel an den 32. Abschnitt von Sein und Zeit in Verbindung mit der in der Vorlesung Was heißt Denken? erschienenen Behauptung, dass jede Übersetzung schon Auslegung ist (vgl. Herrmann, 1994: 308 ff und Heidegger, 2002: 178), bildet F.-W. von Herrmann die Heideggersche „Theorie der Übersetzung“. Hans-Dieter Gondek weist noch auf andere Texte hin, in denen sich verstreute Bemerkungen zum Übersetzen befinden, also die Vorlesungen der dreißiger und vierziger Jahre zu Heraklit und Parmenides, aber auch zu Hölderlin (Gondek, 1996: 41). Gondek kritisiert die von F.-W. von Herrmann geschaffene „Theorie der Übersetzung“, und der Haupteinwand gegen sie ist der, dass F.-W. von Herrmann die Auslegung, die in der Vorlesung Was heißt Denken? erscheint, als die fundamental-ontologische Auslegung von Sein und Zeit versteht. Auf diese Weise wird die Übersetzung hermeneutisch, Gondek protestiert aber dagegen. Der Sprung in den anderen Anfang, d.h. in das Seyn, bricht mit der hermeneutischen Auslegung. Gondek bemerkt richtig: „In Sein und Zeit sowie den Vorlesungen der zwanziger und frühen dreißiger Jahre läßt sich keine explizite Thematisierung des Übersetzens entdecken. Das trifft sogar für die Vorlesung vom Sommersemester 1926 über "Die Grundbegriffe der antiken Philosophie« zu, die, insofern sie unter anderem »die Philosophie vor Plato«, also Heraklit, Parmenides, die Atomisten und die Sophisten behandelt, für eine begleitende Erörterung des Übersetzungsproblems prädestiniert gewesen wäre" (Gondek, 1996: 42). Die Übersetzung als Thema für das Denken gehört zum Entwurf des Seyns. Statt der 
„Theorie der Übersetzung“ gibt Gondek fünf Aspekte an, die auf eine Radikalisierung des Heideggerschen Standpunktes hinweisen (Gondek, 1996: 46-55).

Die Voraussetzungen meines Versuches stehen dem Standpunkt von Gondek näher, weil die hermeneutisch-phänomenologische Interpretation der späten Philosophie von Heidegger mich nicht überzeugt. Ich meine überdies, dass die hermeneutisch-phänomenologische Einsicht in das Sein des Daseins im Entwurf des Seyns und in der ganzen späteren Philosophie von Heidegger, die aus dem Entwurf entspringt, durch die Fuge ersetzt wird. Das Denken des Seyns ist, meiner Meinung nach, nicht mehr hermeneutisch-phänomenologisch, sondern fugig (Kołoczek, 2016: 92-114), und selbst wenn es möglich wäre, die neuen Begriffe der Phänomenologie und der Hermeneutik nach dem Sprung in das Seyn zu rekonstruieren, dann müssten sie zuvor auf den Grund der Fuge des Seyns gedacht werden. Weil die Absicht meiner Überlegungen nicht darin liegt, die Konzeption der Heideggerschen Übersetzung genau zu untersuchen, sondern vielmehr die Figur der Übersetzung des Heideggerschen Denken auf andere Sprache zu skizzieren, deshalb wähle ich nur einen Heideggerschen Kommentar, die Übersetzung betreffend, nämlich Fragmente des Essays Der Spruch des Anaximander, und setze sie mit der Figur der Heimkehr zusammen, die in den Erläuterungen zu Hölderlins Dichtung dargestellt wird. Zwar erscheint in dieser Figur keine Notiz über das Übersetzen, aber diese Figur spielt in der Heideggerschen Übersetzung eine sehr wichtige Rolle.

Der Spruch von Anaximander lautet:

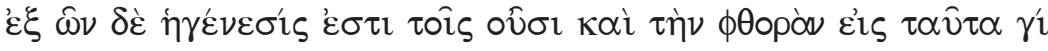

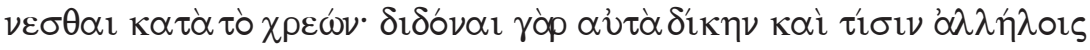

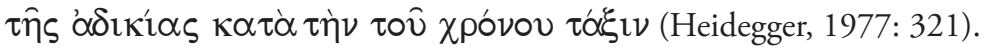

Für meine Ausführung ist es jedoch weder wichtig, was dieses Fragment bedeutet, noch wie es Heidegger genau versteht. Ich werde eher versuchen, mir anzusehen, was er macht, wenn er diesen Text übersetzt. Es wird sich zwar bald herausstellen, dass es nicht leicht ist, den Inhalt und die Übersetzungsart auseinanderzuhalten denn die „Theorie“ der Übersetzung verschmelzt mit dem zu Übersetzenden - dieses Auseinanderhalten muss ich voraussetzen, um das, worum es mir geht, nicht aus den Augen zu verlieren.

Heidegger beginnt zwei zwar unterschiedliche, zugleich jedoch sehr ähnliche Übersetzungen dieses Spruchs ins Deutsche zu zitieren, die eine stammt von F. Nietzsche, die andere von H. Diels. Der erste Zug gehört eher zum Standard und verspricht noch nichts Außergewöhnliches. Die Zusammenstellung beider Übersetzungen mit dem griechischen Text bringt uns auf den Gedanken: „Die Übersetzung von Diels ist in manchem wörtlicher“. Erst diese Feststellung lässt 
Heidegger den Kommentar äußern, der zum Ausgangspunkt für seine Übersetzungskonzeption wird. „Aber solange eine Übersetzung nur wörtlich ist, braucht sie noch nicht wortgetreu zu sein. Wortgetreu ist sie erst, wenn ihre Wörter Worte sind, sprechend aus der Sprache der Sache" (Heidegger, 1977: 322). Diese Unterscheidung zwischen der wörtlichen und der wortgetreuen Übersetzung bedeutet vor allem, die wörtlichen Regeln der Übersetzung außer Kraft zu setzen. Man soll aber nicht nur die Wörterbücher ins Regal zurückstellen, auch die Tradition des Verstehens dieses Spruches, die im Essay nur kurz gefasst wird, sichert nicht den Zugang zu dem, was im Spruch gesagt wird. Um bis zu dem vorzudringen, was dieser Spruch aus sich selbst spricht, scheint es unbedingt notwendig, den historischen Ballast abzulegen. Die Frage ist aber nicht so einfach. „Das bloße Absehen von späteren Vorstellungen führt zu nichts, wenn wir nicht vordem zusehen, wie es mit der Sache steht, die im Übersetzen von einer Sprache in die andere übergesetzt werden soll“ (Heidegger, 1977: 323). Die Übersetzung wäre nur dann wortgetreu, wenn sie aus dem Durchdenken dessen käme, was im Spruch ausgedrückt wurde. „Bei aller Sorgfalt für die philologisch geklärte Sprache müssen wir im Übersetzen zuerst auf die Sache denken“" (Heidegger, 1977: 323). Was ist die Sache, an die man denken muss und die man erst, wenn man nachgedacht hat, übersetzen soll? „Die Sache aber ist hier die Sache des Denkens“ (Heidegger, 1977: 323).

Gerade an dieser Stelle erscheint das grundsätzliche Problem der Heideggerschen Übersetzung. Das In-Frage-Stellen der philologischen Rigorosität, das zur Ablehnung der Äquivalenz der Wörter als Legitimierung der Übersetzung führt, und das In-Frage-Stellen der Tradition als der Gewähr des Zugangs zu dem ursprünglichen Sinn, impliziert die Frage nach einer neuen Rigorosität der Übersetzung. Was Anderes, wenn weder die philologischen Regeln der Übersetzung, noch die seit undenklichen Zeiten überlieferten Auslesungen dieses Spruches, soll einen Raum der möglichen Interpretationen von der gesagten Sache anweisen? Sollte man nicht zuerst richtig verstanden haben, was der Spruch sagt, und dann erst versuchen, diese im Spruch gesagte Sache zu denken? Die Sprache ist doch erst der Zugang zur Sache des Denkens, zuerst muss man also das Griechische von Anaximander verstanden haben, um dann die Sache zu denken (aber in welcher Sprache? Selbstverständlich: auf Deutsch), die Sache, die auch er gedacht hat. Wenn es aber so ist, dann setzt die Forderung von Heidegger, die sagt, dass man die philologische und historische Rigorosität in Frage stellen muss, auch den Zugang zu dieser Sache außer Kraft. Mit anderen Worten: Heidegger bricht hinter sich alle diese Brücken $\mathrm{ab}$, über die er zur Sache hinübergehen könnte. 
Wie ist es möglich an die Sache zu denken, ohne vorher den Spruch übersetzt zu haben, in den sich diese Sache eingesetzt hat?

Die Antwort auf diese Frage sollte eigentlich den ganzen Entwurf der Philosophie des Seyns umfassen. Man kann die kaum im Essay abgezeichnete Diagnose der hermeneutischen Position des Übersetzers mit der Vorlesung der Seinsvergessenheit ergänzen und in die Gestalt des Anklangs einschreiben. Die Auseinandersetzung dagegen, die Heidegger im Essay mit der Aristotelischen Tradition des Verstehens dieses Spruches führt, kann man als das Zuspiel interpretieren. Damit die Übersetzung nach Heideggerschen Regeln möglich ist, ist der Sprung in die Sache des Denkens selbst, d.h. ins Seyn, nötig, aber nicht nur der Sprung, sondern auch das Denken im Sprung, was bei Heidegger die Gründung genannt wird. Sicherlich würde dieses Bild fordern, noch daran zu denken, was in der Sache des Denkens das Letzte ist, d.h. was sich am radikalsten in ihr verbirgt - Heidegger nennt es „der letzte Gott“, im Essay spricht er aber von Eschatologie des Seins. Statt mich auf die komplexe Vorlesung zu konzentrieren, beschäftige ich mich mit ein paar Stellen im Essay, um die Besonderheit der Heideggerschen Übersetzung zu enthüllen. Dennoch bleibt diese Feststellung in Kraft: die Übersetzung ist möglich nur im Rahmen des Entwurfs des Seyns und sie setzt bereits diesen Entwurf voraus. Dieser Entwurf gibt der Übersetzung eine Rigorosität (und das ist die Rigorosität der Fuge). Und die Erklärung des Wesens der Heideggerschen Übersetzung dieses Spruches wirft auch einen klärenden Schein auf den Entwurf des Seyns selbst.

Hier wäre aber Vorsicht angesagt, weil nicht nur die Übersetzung, sondern auch die Auslegung des Spruches von Anaximander im Entwurf des Seyns eingeschrieben ist. Das Ergebnis der Übersetzung, d.h. die Auslesung dieses Spruches, gehört zu dem auf Deutsch gedachten anderen Anfang. Meine Aufgabe besteht aber nicht darin, diese Auslegung genau zu besprechen. Ich möchte bei dem Übersetzungsvorgang selbst anhalten. Zugleich ist es sehr schwierig, diese Abschnitte, die über den Heideggerschen Übersetzungsvorgang berichten, von denen zu unterscheiden, die schon eine Übersetzung sind und das im Spruch Gesagte nennen. Diese beiden Schichten des Textes sind eng miteinander verbunden. Die Frage ist aber komplizierter. Die Übersetzung ist nämlich eine Art des Sich-Übersetzens in das, was denkenswert ist, also sie ist eine Art von Denken; und zugleich hat das, was denkenswert ist, den Charakter der Übersetzung, weil es sich in das Denken übersetzt. Es scheint also einerseits, dass die Sache des Denkens die Übersetzung ist, andererseits ist aber das Denken selbst die Übersetzung.

Ich wiederhole also die Frage: Wie ist es möglich, an die Sache zu denken, bevor der Spruch, in den sich diese Sache eingesetzt hat, übersetzt wurde? 
Die Übersetzung - sagt Heidegger - ist Zwiesprache: „Der Spruch des Denkens läßt sich nur in der Zwiesprache des Denkens mit seinem Gesprochenen übersetzen“ (Heidegger, 1977: 328). Das ist noch eine weitere Feststellung, die die gewöhnliche Denkensart über die Übersetzung anficht. Die Übersetzung ermöglicht doch den Dialog, dieser folgt ihr. Überdies geht es Heidegger nicht um den Dialog mit Anaximander, der einen Satz geäußert hatte. Heidegger kommt ins Gespräch mit dem, worüber der Spruch spricht: „Wir versuchen, den Spruch des Anaximander zu übersetzen. Dies verlangt, daß wir das griechisch Gesagte in unsere deutsche Sprache herübersetzen. Dazu ist nötig, daß unser Denken vor dem Übersetzen erst zu dem übersetzt, was griechisch gesagt ist ${ }^{\text {" }}$. Das griechisch Gesagte muss jetzt auf Deutsch gesagt werden, aber vorher muss es gedacht werden, ähnlich wie zuerst Anaximander es gedacht hatte. Die Zwiesprache von Heidegger mit dem, was der Spruch sagt, ist fast analog zu der Zwiesprache, die Anaximander mit der Sache des Denkens geführt hat. Es gibt nur ein paar Unterschiede. Einerseits kennen wir das „Medium“, durch das die Sache des Denkens Heidegger anspricht - das ist der Spruch von Anaximander. Wir wissen dagegen nichts davon, wodurch sie den griechischen Philosophen angesprochen hat. Auf jeden Fall ist das Ergebnis dieses Dialogs zwischen Anaximander und der Sache des Denkens sein Spruch, der bis heute aufbewahrt ist. Das Ergebnis des Dialogs von Heidegger ist dieser Essay, der den Spruch von Anaximander interpretiert. Ein grundsätzlicher Unterschied besteht aber darin, das Anaximander seine Zwiesprache ins Griechische geführt hat, Heidegger jedoch ins Deutsche. Beide versuchen die Sache des Denkens zu denken, aber beide auf eine andere Weise. „Anders“ heißt hier vor allem: in anderer Muttersprache.

Nachdem die philologische Rigorosität außer Kraft gesetzt worden ist, gibt es keinen unmittelbaren Durchgang zwischen dem griechischen und dem deutschen Text. Der Essay von Heidegger ist also kein philologisches Äquivalent dieses Spruches. Wenn das aber eine gelungene Übersetzung ist, dann weil im Essay dasselbe

\footnotetext{
1 (Heidegger, 1977: 329). Heidegger sagt ausdrücklich: „Wir fragen nicht, ob ǒ v durch »seiend « und ع̂v $\alpha \iota$ durch «sein» richtig übersetzt seien. Wir fragen nur, ob bei diesem richtigen übersetzen auch richtig gedacht werde. Wir fragen nur, ob in dieser geläufigsten aller Übersetzungen überhaupt noch etwas gedacht sei“ (Heidegger, 1977: 333). Die Antwort ist freilich negativ: „Genau besehen, legen wir den griechischen Wörtern nicht einmal eine Bedeutung unter. Wir nehmen sie unvermittelt aus dem ungefähr Verständlichen auf, das ihnen die geläufige Verständlichkeit der eigenen Sprache schon geliehen hat. Wir legen den griechischen Wörtern nichts unter, es sei denn die nachgiebige Fahrlässigkeit eines flüchtigen Meinens." (Heidegger, 1977: 334).
} 
zur Sprache kommt, was im Spruch von Anaximander gekommen ist. Dasselbe, jedoch anders. Dasselbe, aber in anderer Sprache.

Das Zerstören des philologisch-historischen Stegs zwischen Sprachen und dem Verzicht auf das Blättern in Wörterbüchern bedeutet jedoch nicht die Sprache zu verlassen. Die die Übersetzung legitimierende Instanz ist hier weder Mystisch noch Animalisch. Es ist gerade umgekehrt - der Zusammenhang des Denkens und der Sprache, aber nicht der Sprache als solche, sondern der Muttersprache, wird für Heidegger primär. Eben weil Heidegger die Sache des Denkens im Griechischen nicht denken kann, weil das Griechische für ihn die Fremdsprache ist, meint er, dass die philologische Übersetzung den Zugang zur Sache des Denkens nicht sichert, und in diesem Sinne ist sie grundlos. „Wir sind an die Sprache des Spruches, wir sind an unsere Muttersprache, wir sind für beides wesenhaft in die Sprache und in die Erfahrung ihres Wesens gebunden. Diese Bindung reicht weiter und ist strenger, aber auch unscheinbarer als die Maßgabe aller philologischen und historischen Tatsachen, die ihre Tatsächlichkeit von ihr nur zu Lehen haben. Solange wir diese Bindung nicht erfahren, muß jede Übersetzung des Spruches als eine bloße Willkür erscheinen. Doch auch dann, wenn wir durch das Gesprochene des Spruches gebunden sind, behält nicht nur das Übersetzen, sondern sogar die Bindung den Anschein des Gewaltsamen. Gleich als leide das, was hier zu hören und zu sagen ist, notwendig Gewalt" (Heidegger, 1977: 328).

Die Erfahrung der Gewalt der Muttersprache ist für die Heideggersche Philosophie, meiner Meinung nach, wesentlich. Heidegger kann die Sache des Denkens im Griechischen nicht denken, weil er sie im Deutschen denkt. Die Verbindung zwischen dem Denken und der Muttersprache ist so eng, dass man nicht anders denken kann als in der Muttersprache. „In ihm [dem Denken] kommt allem zuvor erst die Sprache zur Sprache, d. h. in ihr Wesen“ (Heidegger, 1977: 328). Das Denken als das Wesen der Sprache lässt die Sprache gerade diese Sprache werden. Zugleich ist das Denken aber nicht etwas von der Sprache Getrenntes, sondern ihr Wesen, d.h. etwas, was sich in der Sprache selbst halten muss. Ich wage folgende Schlussfolgerung: Da im Denken das Deutsche Deutsch wird, dann muss das Denken selbst (d.h. das Denken, an dem Heidegger Anteil hat) das deutsche Denken sein. Analog kann man sagen: Da im Denken das Griechische Griechisch wird, muss das Denken selbst (jetzt d.h. das Denken, an dem Anaximander Anteil hat) das griechische Denken sein. (Dieser zitierte Satz wird im Kontext der Anweisung auf das Denken als ursprüngliches Dichten geäußert und das Denken selbst wird weder nur auf die Heideggersche Philosophie, noch auf die Philosophie als solche beschränkt, sondern bezieht sich auf jedes Schaffen: „Alles Dichten in diesem 
weiteren und im engeren Sinne des Poetischen ist in seinem Grunde ein Denken“ (Heidegger, 1977: 329)).

Die Übersetzung muss also - ins Deutsche - über die Sache des Denkens berichten, aber auch darüber, warum diese Sache im Spruch von Anaximander auf solche Weise artikuliert wurde. In diesem Sinne behält die Übersetzung die Verbindung zu dem Original. Ein solches Klarstellen dieser Angelegenheit bedeutet jedoch, dass Heidegger der Verbindung mit den Wörtern nicht entsagt. Seine ganze Arbeit erfolgt in der Materie des Wortes, was jedem, der zu seinem Essay greift, klar wird. Diese in Wörterbüchern vorkommenden Wörter erscheinen als ausgesprochen wichtig. Der hier schon angeführte Abschnitt sagt, dass man diese Wörter als „sprechend aus der Sprache der Sache" hören soll. Ein bisschen weiter

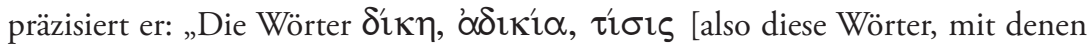
der Spruch von Anaximander ausgedrückt wurde - ŁK] haben keine fachlich eingegrenzte, sondern eine weite Bedeutung. Weit meint hier nicht: ausgeweitet, verflacht und verdünnt, sondern weitreichend, reich und Vorgedachtes bergend. Darum allein und darum gerade sind jene Wörter geeignet, das mannigfaltige Ganze im Wesen seiner einigen Einheit zur Sprache zu bringen. Damit das geschehe, muß freilich das einige Ganze des Mannigfaltigen mit seinen eigenen Zügen rein an ihm selbst im Denken vernommen sein" (Heidegger, 1977: 331f). Die Figur, nach der der Gedanke aufgestellt ist, soll durchdacht werden. Die Wörter - die nur nach der Wörterbuchorthodoxie nicht übersetzt werden sollten - bergen in sich etwas, was schon früher als das Gedachte selbst war. Dieses Vorgedachte ist freilich die Sache des Denkens, die von Heidegger identifiziert wird als das Sein des Seienden im Ganzen, d.h. als mannigfaltige Ganzheit des Seienden. In den Wörtern kann diese mannigfaltige Ganzheit zur Sprache gebracht werden, aber nur wenn das Denken diese Ganzheit an sich vernimmt. Die wörtliche Übersetzung also, das heißt solche, die die Wörter berücksichtigt, jedoch das übersieht, was sich in ihnen verbirgt, verfehlt das, worum es im Spruch geht. Die Übersetzung, die auf die Sache des Denkens gerichtet ist, kann sich nicht damit begnügen, was in den Wörtern an den Tag kommt, sondern sie muss Rücksicht darauf nehmen, was sich in der Helligkeit der Wörter verbirgt.

Die wichtigsten Wörter sind selbstverständlich عîv $\alpha$ l und „das Sein“. Die durch Wörterbücher und Tradition bestimmte Übersetzungsmöglichkeit des Einen ins Andere ist keine Möglichkeitsbedingung, die Geschichte der Philosophie zu betreiben, sondern eher die Konsequenz dessen, dass alle Weisen des Denkens, die unsere philosophische Tradition bauen, sich in einem „Bereich aller Bereiche“ befinden, der durch Wörter E̊̂v $\alpha \mathrm{l}$ und ǒ $v$ bestimmt ist. An dieser Stelle wiederholt 
sich die Figur der Heideggerschen Philosophie: wir wissen gewöhnlich, was diese Wörter bedeuten. „Meist legen wir unbedenklich den Wörtern ő $v$ und عiv $\alpha_{1}$ das unter, was wir selbst bei den entsprechenden Wörtern unserer Muttersprache an Ungedachtem meinen: Seiendes und Sein" (Heidegger, 1977: 334). Was wir von ihren Bedeutungen wissen, ist uns wahrscheinlich im Alltag genug: „Genau besehen, legen wir den griechischen Wörtern nicht einmal eine Bedeutung unter. Wir nehmen sie unvermittelt aus dem ungefähr Verständlichen auf, das ihnen die geläufige Verständlichkeit der eigenen Sprache schon geliehen hat. Wir legen den griechischen Wörtern nichts unter, es sei denn die nachgiebige Fahrlässigkeit eines flüchtigen Meinens. Dies mag zur Not hingehen, wenn wir z. B. Évvol und

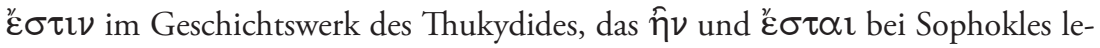
sen" (Heidegger, 1977: 334). Wenn wir jedoch uns das anschauen, was sich in den Wörtern verbirgt, eröffnet sich die Möglichkeit der philosophischen Übersetzung.

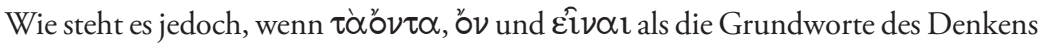
und nicht nur eines beliebigen Denkens, sondern als das Hauptwort des ganzen abendländischen Denkens in der Sprache laut werden? Dann offenbart eine Prüfung des übersetzenden Sprachgebrauches folgende Sachlage:

Weder ist klar und gegründet, was wir selbst bei den Wörtern der eigenen Sprache "seiend " und "sein" denken;

noch ist klar und gegründet, ob das jeweils von uns Gemeinte das trifft, was die Griechen in den Wörtern ő $\nu$ und Ei $v \propto l$ ansprechen.

Weder ist klar und gegründet, was denn überhaupt ơ $\nu$ und عîv $\alpha$ l, griechisch gedacht, sagen;

noch läßt sich bei dieser Sachlage jemals eine Prüfung anstellen, ob und inwieweit unser Denken dem der Griechen entspricht.

(Heidegger, 1977: 334f.)

Diese „Verwirrtheit“, der der abendländische Mensch seit Jahrtausenden unterworfen ist, ist nicht nur Ergebnis der Defizite. „Sie kommt aus dem Abgrund des Verhältnisses, worein das Sein das Wesen des abendländischen Menschen ereignet hat" (Heidegger, 1977: 335).

Die Übersetzung wird erst dann gelingen, wenn zwei Bedingungen erfüllt werden. Erstens: die griechischen Wörter werden den Übersetzer auf diese mannigfaltige Ganzheit lenken, auf das (sich) Zuteilen des Seins, das sich in das archaische Griechenland geschickt hat, das heißt, wenn es den Übersetzer gelingen wird, in das, was sich im Griechischen des Spruches von Anaximander verbirgt, zu springen. Zweitens: wenn es ihm gelingen wird, die deutschen Wörter so zu überlegen, dass 
sie das neue Zuteilen aufbewahren können, solches Zuteilen, das das Sein dem deutschen Volk gewährt.

Diese sehr ähnliche Figur, obwohl mit der Übersetzung nicht so klar verbunden, wird im Heideggerschen Kommentar zu dem Gedicht Heimkunft/An die Verwandten von Hölderlin sichtbar. Die Reise in fremde Länder füllt das Herz des Wanderers mit Nostalgie, aber sie macht ihn „erfahrener“ („Der heimkommende Wanderer ist im Wesen der Götter, d.h. der Freudigen, erfahrener geworden “ [Heidegger, 1981: 20]). Die Heimkunft ist zwar „die Rückkehr in die Nähe des Ursprungs“, sie bedeutet aber keines Falls das Ende der Wanderung, sondern erst ihren Anfang. Der durch die Reise Erfahrene macht sich, zurückkehrend, erneut auf die Wanderung zum Wesen des Heimischen. Die Rückkehr nach Hause lässt erkennen, dass das gut Gekannte und durch alltägliche Sorgen Vertraute das Wesen des Heimischen nicht ausschöpft, dass das Heimische die Offenbarung seines Wesens verweigert („Die Nähe zum Ursprung ist eine sparende Nähe. (...) Im Wesen der Nähe ereignet sich ein verborgenes Sparen“ [Heidegger, 1981: 24]). Die Struktur, die Heidegger in seiner Erörterung rekonstruiert, ist um das besondere Verstehen der Gabe geordnet. Im gewöhnlichen Sinne gehört der Wanderer zu dem Heimischen und seine Heimat gehört zu ihm. Wenn aber dieser Wanderer Hölderlin, der Dichter, ist, dann wird dieses einfache Verhältnis des Besitzens durchbrochen: „Doch in der Schickung ist das Eigene gleichwohl noch nicht übereignet“ (Heidegger, 1981: 14). Das heißt, dass das Eigenste der Heimat kein Eigentum der Bewohner ist. Es versagt sich ihnen. Die Gabe, die jeder Landsmann und jede Landsfrau bekommt, wenn er oder sie geboren werden, stellt sich in der Erfahrung des Dichters heraus als die Gabe, die sich aufspart und zu weiterer Wanderung einlädt. Das Eigene „wird noch zurückbehalten“ (Heidegger, 1981: 14). Die Gabe - dichterisch gedacht ist das Sichverweigern der Gabe selbst. „Was du suchest, es ist nahe, begegnet dir schon“ (Heidegger, 1981: 13) „aber das Beste, der Fund, (...) ist Jungen und Alten gespart“" (Heidegger, 1981: 14).

An dieser Stelle des Heideggerschen Kommentars besteht kein Zweifel darüber, was das Wesen der Heimat ist. Das ist das Deutsche. Heidegger notiert, dass der Wortlaut dieses Gedichtes geändert wurde, und dass Hölderlin in einer späteren Variante statt der Phrase „aber das Beste, der Fund“ geschrieben hat: „Aber der Schatz, das Deutsche ... ist noch gespart“.

Die Heimkehr, die zu der Struktur des Platonischen Mythos über die Höhle isomorph ist, ist die Rückkehr aus der Fremde. Für Hölderlin bedeutet die Fremde 
Griechenland ${ }^{2}$. Es scheint mir, dass eben dieser Moment Heidegger angezogen hat: sich auf den Weg nach Griechenland machen wie in eine Emigration, „erfahrener“ werden nicht nur in dem Wesen der Götter, sondern vor allem auch in dem Wesen des Seins. Der Aufenthalt in Griechenland, d.h. der Blick gerade in die Sonne, nimmt erst dann jedoch Sinn an, wenn er die Rückkehr ermöglicht. Die Rückkehr bedeutet hier den Weg zum Deutschen, zu dem, was sich den Deutschen verweigert.

Genau dieselbe Struktur hat die Übersetzung, die Heidegger betreibt: Man soll zu dem, was im Griechischen gedacht wurde, gelangen, mit dem „Eingenommen“, zum Deutschen zurückkehren, um dieses im Griechischen gedachte Wesen jetzt im Deutschen zu äußern. Das Gedachte - die Sache des Denkens, das Wesen des Seins - verbirgt sich sowohl in den im Griechischen geschriebenen Sätzen als auch in den Sätzen, die Heidegger auf Deutsch artikuliert. Es verbirgt sich und auf diese Weise ruft es zur Ausdauer auf dem Weg zu diesem Wesen auf. Dank dieser Übersetzung ereignet sich aber etwas Ungewöhnliches. Das Deutsche, das in die Worte der Hölderlinschen Dichtung und die Worte der Heideggerschen Philosophie gehüllt ist, spart sich selbst als eine geschichtliche Gestalt des Wesens der Metaphysik. Diese Übersetzung zeigt sich als die Übersetzung des Wesens der Metaphysik aus dem Bereich, wo das Griechische dem griechischen Philosophen gespart ist, in den Bereich, wo das Deutsche sich dem deutschen Philosophen verbirgt.

An dieser Stelle möchte ich die Frage stellen, wie jemand, dessen Muttersprache weder Griechisch noch Deutsch ist, die Philosophie von Heidegger lesen sollte. Ich denke dabei nicht an die Schwierigkeiten, die ein Fremdsprachiger haben könnte, sowohl dem Sprachgefühl der Heideggerschen Philosophie zu folgen, als auch das Heideggersche Idiom zu entziffern. Die Sache liegt tiefer, d.h. im Eintauchen jedes Menschen, insbesondere eines Philosophen, in die eigene Muttersprache. Es geht darum, wann der Kommentator der Heideggers Philosophie bei sich selbst ist. Wenn wir hier mit der Metapher einverstanden sind, die zwar ein Gedankenkürzel, aber keine Verfälschung des Heideggerschen Entwurfs ist, und besagt, dass das Denken des Seyns das zu sich Kehren bedeutet, aber so zu sich kehren, dass das gesparte Wesen des Selbst geschützt und in sich aufbewahrt wird, dann wird es klar, dass

2 Im Kommentar zum Gedicht Andenken setzt Heidegger die Hölderlinsche Reise in den Süden Frankreichs mit der Reise in die Fremde gleich, um das Wesen Griechenlands kennen zu lernen. Unter Bezugnahme auf den Hölderlinschen Brief an seinen Freund, Böhlendorf, notiert Heidegger: „Im zweiten Brief schreibt Hölderlin, das Menschentum des südlichen Frankreichs habe ihn mit dem eigentlichen Wesen der Griechen bekannter gemacht. Das Verweilen in der Fremde des südlichen Himmels bringt dem Dichter zum voraus und stets eine höhere Wahrheit in sich: das "Denken« dieses Dichters "an« das Land der Griechen" (Heidegger, 1981: 82). 
das Denken des Seyns etwas Anderes für einen deutschen Philosophen (oder einen Kommentator dieser Philosophie - hier wird das Denken des Seyns immer mit der Einstellung zu deutscher Sprache und deutschem Volk zusammenhängen) bedeutet und etwas Anderes für einen nicht Deutschsprachigen. Um die allgemeinen Sätze und die Versuchung der Äußerung im Namen aller nicht Deutschsprachigen zu meiden, berufe ich mich auf meine Erfahrung mit Lektüre der Beiträge zur Philosophie. Die Figur der Heimkehr, die erst mit dem Weg zu dem sich gesparten Wesen der Heimat beginnt, bedeutet in diesem Fall nicht den Weg zum Wesen des Deutschen, sondern zum Wesen des Polentums. Ich bin in dieser Sprache eingewurzelt, ich denke in Polnisch, selbst wenn ich Heidegger lese.

Das, worin das Wesen des Polentums besteht, ist seit Jahren das Thema des Streites unter Polen. Viele bedeutende nationale Philosophen haben über diese Frage nachgedacht. Der Zank über das Polentum bildet unsere ganze Geschichte und Mythologie, er durchdringt die polnische Literatur und Politik, unsere Leidenschaften und Wahlen, er verursacht, dass wir uns Träumereien hingeben und doch auf dem festen Boden der Tatsachen bleiben. Unsere Sprache ist von diesen Streitigkeiten um die Historiographien und Mythologien gesättigt. Wir sind Polen, indem wir uns darum streiten, was es bedeutet, ein Pole zu sein. Wir stecken in dieser Unentschiedenheit, ob wir im Westen die Armen oder im Osten die Herren sein sollen (vgl. Janion, 2007). Die Heimkehr ist also nicht die Rückkehr zum heimischen Hafen, wo Landsleute ihren alltäglichen Tätigkeiten nachgehen. Sie bedeutet eher die Rückkehr in die Unbestimmtheit, in die Ungewissheit der Existenz des Volkes.

Wenn die Lektüre des Entwurfs von Heidegger das Denken des Seyns sein sollte, d.h. die Heimkehr, dann muss die Lektüre auf Polnisch das Denken im Polnischen bedeuten, die Rückkehr zum Polentum, das Warten auf sein Wesen, das Bergen der Schickung des Seyns, das sich in dieser Sprache ereignet. Die fundamentale Frage der Beiträge zur Philosophie - wer wie sind? (vgl. Heidegger, 1989: 44, 48-54, 125 ) - ist eben deshalb unübersetzbar, weil dieses „sind“ eine andere Zuweisung des Seyns ist als das polnische ,jesteśmy“ (d.h. das polnische Wörterbuchäquivalent des Deutschen „sind“).

Heidegger erfährt auf dem Weg zum Deutschen das Fremde, und das ist für ihn Griechenland. Das Fremde für einen Polen, so fremd bis zur Stummheit (niemy), ist das Deutsche (niemieckość). Darüber belehrt uns die Sprache, nicht unbedingt die existenzielle Erfahrung. Ich meine, dass eine der Arten das Seyn zu erfahren, das sich im Polnischen ereignet und in ihm verbirgt, die Übersetzung des Entwurfs von Heidegger ins Polnische ist. Es kann aber selbstverständlich keine wörtliche 
Übersetzung sein. Die Werke von Heidegger werden ständig ins Polnische übersetzt und diese Arbeit eines ganzen Stabes von Philosophen, unter denen man Janusz Mizera erwähnen sollte, hat mit Sicherheit die polnische philosophische Sprache verändert. Die übersetzerische Arbeit ist freilich außergewöhnlich wichtig und sinnvoll. Das Problem liegt jedoch darin, dass solche Übersetzungen - aus, sagen wir, objektiven Gründen wie: Anforderungen der Verleger oder Erwartungen der Leser - dem Original treu sein müssen. Im Bereich der Worttreue kann man selbstverständlich bessere und schlechtere Übersetzungen finden, denn der Übersetzung entgeht immer etwas, was im Original gestanden hat, und etwas Neues taucht in ihr auf. Im Falle der Beiträge zur Philosophie kann aber die Treue zu dem Original nicht in Wortgetreulichkeit bestehen, sondern eher in etwas ganz Anderem. Die Übersetzung soll sich nicht darum kümmern, was die Worte ausdrücken und ausdrückend offenbaren, sondern sie soll eher darauf Acht geben, was sich dem Deutschen, folglich auch den deutschen Wörtern, verweigert und sich auf diese Weise in ihren verbirgt. Diese das Seyn bergende Stärke des abgenutzten Wortes soll in die andere Sprache übersetzt werden. Aber das bedeutet: sie muss jetzt neu erfahren werden, anders, (in meinem Fall) im Polnischen. Hier erscheint eben das Problem. Im Original ist das Deutsche in Deutsch und auf deutsche Weise aufbewahrt. Die Übersetzung kann keine von diesen drei Momenten der Bergung erhalten. Wenn sie das Erhalten der Aufbewahrung sein sollte, dann müsste sie sich als die Aufbewahrung des Polentums im Polnischen auf polnische Weise ereignen. Die wesentliche Übersetzung muss etwas ganz Anderes als nur Übersetzende sein.

Das Wort „Übersetzung“ wird jetzt zweideutig. Diese Zweideutigkeit hat ihren Ursprung in der Gebärde von Heidegger selbst im Text Anaximanders Spruch (mehrmals noch in anderen Texten wiederholt), der die philologischen Übersetzungen von Nietzsche und Diels verlässt und eine neue Art vorschlägt. Um Missverständnisse, die der Zweideutigkeit entspringen, zu meiden - zum Beispiel den Verdacht, dass ich eine neue Edition der Übersetzungen von Heideggerschen Werken ins Polnische verlangen würde oder jemandem die Vorbereitung neuer Übersetzungen verleiden möchte - zugleich um die in den Wörtern „übersetzen “ und „przekładać” inbegriffenen Intuitionen zu erhalten, schlage ich vor, diese Heideggersche Übersetzung „Transposition“ zu nennen. Das Wort bezeichnet dieselbe Struktur - nämlich die Versetzung. Das Präfix des Wortes „Transposition“, also „trans“, bedeutet aber vor allem die Stellungnahme zwischen dem Deutschen des Entwurfs von Heidegger also zwischen etwas Fremdem - und dem Polnischen als „Eigentum“ des Übersetzers, das man erst dann vernehmen soll als Schickung des Seyns. 
Die Transposition (als die Form des Denkens an das Seyn im Polnischen) wird, indem sie die Struktur der Rückkehr zu sich selbst - zum Polentum erhält, „erfahrener" durch das Nachdenken über das Fremde, d.h. das Deutsche. Das zu sich Kommen wird dann zum Gang durch Beiträge zur Philosophie. Wenn die Rückkehr aus der Verbahnung in Beiträge zur Philosophie gelingt, dann wird die Travestie der Heideggerschen Feststellung möglich: im Entwurf des (im Polnischen gedachten) Seyns gibt es nichts Deutsches mehr (Heidegger, 1986: 366).

Das Anderssein des im Polnischen gedachten Entwurfs des Seyns ist jedoch nicht absolut. Das Seyn verbirgt sich ebenso, wenn es im deutschen Element gedacht ist, als wenn es in das Polnische eingetaucht wird. Die Verborgenheit des Seyns kann in keiner Sprache offenbart werden. Sie kann jedoch in dem Offenbarten aufbewahrt werden. Das Deutsche und das Polnische hüten beide auf ihre eigene Weise das Seyn. Dank Heidegger weißt man, wie sich das ereignen soll, nämlich in der Form der Fuge. Damit der im Polnischen gedachte Entwurf des Seyns die Verborgenheit aufbewahrt, muss er auch seine Fuge offenbaren. Das ist die übergeordnete Aufgabe der Transposition - die fugische Struktur des Polnischen auf solche Weise zu entwerfen, dass man alles, was sich in dieser Sprache äußern lässt, zur Verfügung dessen stellen kann, was sich dem Offenbarten verweigert.

Die Absicht die Fuge im Polnischen zu entwerfen, basiert auf der fundamentalen Voraussetzung, nämlich auf der Fugigkeit des Seyns selbst ${ }^{3}$. Heidegger bahnt sich den Zugang zum Seyn, indem er im Deutschen heimisch wird. Damit offenbart er den Volkscharakter seiner Fuge. Es muss so sein, wenn das als die Behausung des Seyns erfahrene Deutsche das Seyns selbst aufbewahrt. Das auf diese Weise aufbewahrte Seyn wird zum Aufbewahrten in dem besonderen Offenbaren. Das Seyn weist sich (in der Art der Verweigerung) solcher Aufbewahrung zu, die, obwohl sie selbst die Form der Fuge hat, kaum die Fügung des Seyns ist, d.h. eine der Einzelteile der Fuge. Das Seyn kann sich - das ist die kardinale Voraussetzung meines Versuchs - auch in eine andere Sprache schicken und sich in die Fuge auf andere Weise einordnen. Die Fugigkeit des Seyns bedeutet also auch, dass die deutsche Fuge mit der polnischen Fuge eine Ganzheit bilden kann, die die Struktur der Fuge haben wird. Es gibt in Wahrheit keine übergeordnete Sprache, in der diese Ganzheit artikuliert werden könnte, aber es gib die Transposition, die sich aus einer in andere Fuge legen lässt, d.h. aus einer Sprache in die andere, d.h. aus einer Weise der Aufbewahrung zur anderen.

3 Vgl. Heidegger, 1977: 366: „(...) denn das Sein spricht in der verschiedensten Weise überall und stets durch alle Sprache hindurch". 
Die Formulierung dieser Voraussetzung äußert die Überzeugung, dass das Seyn „eins“ „ist“, obwohl es auf vielfältige Weise die Sprachen beherrscht. Die Einheit des Seyns ist keine höchste Allgemeinheit - nota bene das Ordnen der Worte in weiteren Stufen der Allgemeinheit ist dafür blind, in welcher Sprache es gemacht wird: „der Baum des Porphyrios“ verliert von seinem Wesen nichts von dem, was in dem Gelangen an den allgemeinsten Sinn des Wortes „Sein“ besteht, wenn es im Griechischen, Lateinischen, Deutschen oder Polnischen ausgesprochen wird. Das Seyn verfügt inzwischen über die Sprache, in die es sich schickt. Die fugische Identität der deutschen und polnischen Entwürfe - die Identität, die die Transposition zugleich voraussetzt und festlegt, d.h. entwirft - entspringt dem unermesslichen Reichtum des Seyns. Wahrscheinlich kann man die Möglichkeiten nicht aufbrauchen, auf welche das Seyn den Menschen beschenken kann. Die Transposition legt die Verhältnismäßigkeit zwischen zwei Fügungen des Seyns, dem Deutschen und Polentum, und damit eröffnet sie neue Dimensionen des Seyns.

Die Hauptaufgabe der Transposition ist jedoch der Entwurf eines solchen Verständnisses des Polnischen, das den fugischen Charakter dieser Sprache begreifen und bei dem in ihr Verborgenen verhalten wird.

\section{Zitierte Werke}

Gondek, Hans-Dieter. „Das Übersetzen denken: Übersetzen und Übersetzen“. Heidegger Studien 12 (1996): 37-55.

Heidegger, Martin. »Andenken«. Martin Heidegger. Gesamtausgabe, Bd. 4: Erläuterungen zu Hölderlins Dichtung. Frankfurt am Main: Vittorio Klostermann, 1981. 79-151.

----. »Heimkunft / An die Verwandten«. Martin Heidegger. Gesamtausgabe. Bd. 4: Erläuterungen zu Hölderlins Dichtung. Frankfurt am Main: Vittorio Klostermann, 1981. 9-31.

----. Gesamtausgabe, Bd. 65: Beiträge zur Philosophie (vom Ereignis). Frankfurt am Main: Vittorio Klostermann, 1989.

----. „Der Spruch des Anaximader“. Martin Heidegger. Gesamtausgabe, Bd. 5: Holzwege. Frankfurt am Main: Vittorio Klostermann, 1977. 321-374.

----. Gesamtausgabe, Bd. 15: Seminare. Frankfurt am Main: Vittorio Klostermann, 1986.

----. „Vom Wesen und Begriff der Fisis. Aristoteles, Physik B, 1“. Heidegger, Martin. Gesamtausgabe, Bd. 9: Wegmarken. Frankfurt am Main: Vittorio Klostermann, 1976. 239-302.

---. Gesamtausgabe, Bd. 8: Was heißt Denken. Frankfurt am Main: Vittorio Klostermann, 2002. 
Herrmann von, Friedrich-Wilhelm. Wege ins Ereignis. Zu Heideggers „Beiträgen zur Philosophie“. Frankfurt am Main: Vittorio Klostermann, 1994.

Janion, Maria. Niesamowita Stowiańszczyzna. Kraków: Wydawnictwo Literackie, 2007. Kołoczek, Łukasz. Być, czyli mieć. Próba transpozycji projektu „Przyczynków do filozofii” Martina Heideggera. Kraków: Universitas, 2016.

\title{
Transposition as the Way of Reading Contributions to Philosophy
}

\begin{abstract}
Summary
In this article I formulate the problem of translation Heidegger's project of Beyng into other languages, particularly into Polish. Firstly, I note that Heidegger himself makes translation. I investigate what his practice of translation consist of. Secondly, it appears that structurally the translation corresponds to the project of Beyng, which means that it is only possible to carry out within the framework of this project, but at the same time the project is, in one sense, the "result" of this translation. Thirdly, I show that to the essence of this philosophy belongs the language, in which one thinks, and not only for the philological reasons, but primarily because of the relationship of Beyng with speech and speech with thinking. In this context I put the question about the possibility of thinking of Beyng in different language that the German. I suggest to repeat the same gesture of Heidegger, meaning to translate the project of Beyng to Polish in the same way in which he translates from Greek to German. This method I call transposition.
\end{abstract}

Keywords: transposition, translation, Martin Heidegger, Contributions to Philosophy

Słowa kluczowe: transpozycja, przekład, Martin Heidegger, Przyczynki do filozofi 